\title{
Calpain inhibition improves collateral-dependent perfusion in a hypercholesterolemic swine model of chronic myocardial ischemia
}

\author{
Ashraf A. Sabe, MD, ${ }^{a}$ Brittany A. Potz, MD, ${ }^{\mathrm{a}}$ Nassrene Y. Elmadhun, MD, ${ }^{\text {a }}$ Yuhong Liu, MD, ${ }^{\mathrm{a}}$ \\ Jun Feng, MD, PhD, ${ }^{a}$ M. Ruhul Abid, MD, PhD, ${ }^{a}$ Jinnette D. Abbott, MD, ${ }^{a}$ Donald R. Senger, PhD, ${ }^{b}$ and \\ Frank W. Sellke, MD
}

\begin{abstract}
Purpose: Calpain overexpression is implicated in aberrant angiogenesis. We hypothesized that calpain inhibition (MDL28170) would improve collateral perfusion in a swine model with hypercholesterolemia and chronic myocardial ischemia.

Methods: Yorkshire swine fed a high cholesterol diet for 4 weeks underwent surgical placement of an ameroid constrictor to their left circumflex coronary artery. Three weeks later, animals received no drug, high cholesterol control group $(\mathrm{n}=8)$; low-dose calpain inhibition $(0.12 \mathrm{mg} / \mathrm{kg} ; \mathrm{n}=9)$; or high-dose calpain inhibition $(0.25 \mathrm{mg} / \mathrm{kg} ; \mathrm{n}=8)$. The heart was harvested after 5 weeks.
\end{abstract}

Results: Myocardial perfusion in ischemic myocardium significantly improved with high-dose calpain inhibition at rest and with demand pacing $(P=.016$ and .011). Endothelium-dependent microvessel relaxation was significantly improved with low-dose calpain inhibition $(P=.001)$. There was a significant increase in capillary density, with low-dose calpain inhibition and high-dose calpain inhibition $(P=.01$ and .01$)$, and arteriolar density with low-dose calpain inhibition $(P=.001)$. Calpain inhibition significantly increased several proangiogenic proteins, including vascular endothelial growth factor $(P=.02)$, vascular endothelial growth factor receptor $1(P=.003)$, vascular endothelial growth factor receptor $2(P=.003)$, and talin, a microvascular structural protein $(P=.0002)$. There was a slight increase in proteins implicated in endothelial-dependent (nitric oxide mediated) relaxation, including extracellular signal-regulated kinase, phosphorylated extracellular signal-regulated kinase, and inducible nitric oxide synthase with calpain inhibition.

Conclusions: In the setting of hypercholesterolemia, calpain inhibition improved perfusion, with a trend toward increased collateralization on angiography and increased capillary and arteriolar densities in ischemic myocardium. Calpain inhibition also improved endothelium-dependent microvessel relaxation and increased expression of proteins implicated in angiogenesis and vasodilatation. (J Thorac Cardiovasc Surg 2016;151:245-52)

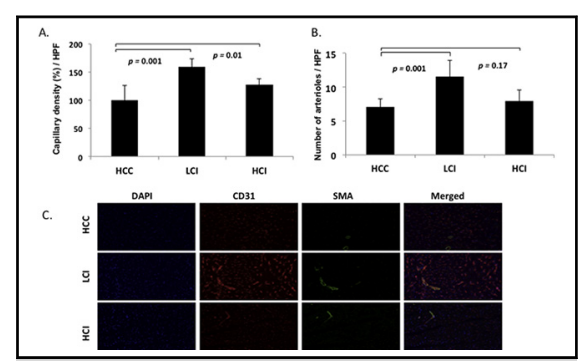

Vessel density was significantly increased in the pigs with calpain inhibition versus controls.

\section{Central Message}

Calpain inhibition in the setting of chronic myocardial ischemia improves proangiogenic protein expression and myocardial perfusion.

\section{Perspective}

Moderate calpain inhibition in the setting of hypercholesterolemia and chronic myocardial ischemia improves proangiogenic protein expression, microvascular relaxation, and myocardial perfusion. These findings may have important clinical implications for the treatment of patients with severe coronary artery disease and microvascular dysfunction resulting from hypercholesterolemia.

See Editorial Commentary page 253.
Despite advances in percutaneous and surgical interventions in the treatment of coronary artery disease (CAD), up to one third of patients are not candidates for or receive

\footnotetext{
From the ${ }^{\text {a Division }}$ of Cardiothoracic Surgery, Cardiovascular Research Center, Warren Alpert School of Medicine, Brown University, Providence, RI; and ${ }^{\mathrm{b}}$ Division of Pathology, Beth Israel Deaconess Medical Center, Harvard Medical School, Harvard University, Boston, Mass.

Funding for this research was provided by the National Heart, Lung, and Blood Institute (R01HL46716, R01HL69024 to FWS), National Institutes of Health (NHI) Training Grant 5T32-HL094300-03 (AAS and NYE), NIH Centers of Biomedical Research Excellence Grant 5P20 GM1P20GM103652 (Project-3, JF and MRA), American Heart Association Grant-in-Aid 14GRNT20460291
}

suboptimal revascularization with these therapies. ${ }^{1}$ The incidence of incomplete revascularization in patients with severe CAD who undergo surgical intervention is an

(MRA), Rhode Island Foundation-RIF-20123834 (JF), and NIH/National Institute of General Medical Sciences Training Grant 2T32 GM065085-11A1 (BAP).

Received for publication July 1, 2015; revisions received Aug 14, 2015; accepted for publication Aug 26, 2015; available ahead of print Oct 15, 2015.

Address for reprints: Frank W. Sellke, MD, Division of Cardiothoracic Surgery, Cardiovascular Research Center, Warren Alpert Medical School of Brown University, 2 Dudley St, MOC 360, Providence, RI 02905 (E-mail: fsellke@lifespan.org). $0022-5223 / \$ 36.00$

Copyright (c) 2016 by The American Association for Thoracic Surgery http://dx.doi.org/10.1016/j.jtcvs.2015.08.101 


$$
\begin{aligned}
& \text { Abbreviations and Acronyms } \\
& \text { CAD }=\text { coronary artery disease } \\
& \text { CI }=\text { calpain inhibition } \\
& \text { ERK }=\text { extracellular signal-regulated kinase } \\
& \text { HCC }=\text { high cholesterol control } \\
& \text { HCI }=\text { high-dose calpain inhibition } \\
& \text { iNOS }=\text { inducible nitric oxide synthase } \\
& \text { LCI }=\text { low-dose calpain inhibition } \\
& \text { LCx }=\text { left circumflex artery } \\
& \text { pERK }=\text { phosphorylated extracellular } \\
& \text { signal-regulated kinase } \\
& \text { RhoA }=\text { ras homolog gene family member A } \\
& \text { VEGF }=\text { vascular endothelial growth factor } \\
& \text { VEGFR1 }=\text { vascular endothelial growth factor } \\
& \text { receptor } 1 \\
& \text { VEGFR2 }=\text { vascular endothelial growth factor } \\
& \text { receptor } 2
\end{aligned}
$$

independent predictor for operative and perioperative morbidity and mortality. ${ }^{2,3}$ With an increased prevalence of obesity and metabolic syndrome, the incidence of severe $\mathrm{CAD}$ not amenable to surgical treatment is likely to increase. ${ }^{1,4,5}$ Inducing angiogenesis through medical therapies remains a promising therapeutic option for these patients. However, a deeper understanding of the proangiogenic and antiangiogenic pathways in the setting of hypercholesterolemia and chronic ischemic disease is necessary to treat this complicated and growing population of patients. Our laboratory has created a pig model for chronic myocardial ischemia in the setting of metabolic syndrome (weight gain, glucose intolerance, dyslipidemia, and hypertension). ${ }^{4}$

Calpains, calcium-dependent thiol proteases expressed ubiquitously in mammals, are an important potential mediator of these angiogenic pathways. When activated, calpains regulate a broad spectrum of functionally important protein targets that involve cytoskeletal organization, cell adhesion, and cell migration. Hypoxia is known to induce calpain activity, resulting in disruption of cardiac endothelial cell cytoskeletal structure and function..$^{6-10}$ Modest suppression of calpain activity has been shown to improve functional neovasculature. ${ }^{7,8}$ Although the mechanism for this improvement remains largely unknown, there is evidence in small animal (rodent) models that calpain inhibition (CI) allows for upregulation of proangiogenic proteins and scaffolding proteins that are essential for new vessel growth and maturation. ${ }^{7,8}$ Although these studies are promising, they have been performed only in small, otherwise healthy animal models. Given the considerable potential for the proangiogenic effects of $\mathrm{CI}$, we sought to investigate their effects in a clinically relevant porcine model of metabolic syndrome. We hypothesized that in the setting of chronic myocardial ischemia, CI would result in improved collateral-dependent myocardial perfusion and vascular function.

\section{MATERIALS AND METHODS \\ Animal Model and Surgical Interventions}

Juvenile male Yorkshire swine (Parsons Research, Amherst, Mass) were divided into 3 groups, fed a high cholesterol diet for 4 weeks, and then underwent surgical placement of a titanium ameroid constrictor (Research Instruments SW, Escondito, Calif) on the proximal left circumflex artery (LCx). Male pigs were used in an effort to limit variables (male vs female) between pigs. Three weeks later, animals received no drug, high cholesterol control (HCC) group $(\mathrm{n}=8)$; an oral form of a low-dose CI (LCI) $(0.12 \mathrm{mg} / \mathrm{kg} ; \mathrm{n}=9)$; or an oral form of a high-dose CI (HCI) $(0.25 \mathrm{mg} / \mathrm{kg} ; \mathrm{n}=8)$ (CI MDL28170; EMD Millipore, Danvers, Mass). The diets and oral form of the CI were continued for 5 weeks until completion of the study, and then the animals were anesthetized and underwent $\mathrm{x}$-ray coronary angiography. The heart was then exposed through a midline sternotomy, and microspheres were injected at rest and with ventricular pacing (160 beats/min). The animals were euthanized, and their hearts were harvested. Tissue samples from chronically ischemic myocardium (LCx territory) and nonischemic myocardium were rapidly frozen in liquid nitrogen. Tissue samples for microvessel reactivity studies were placed in Krebs solution. Detailed methods on surgical procedures, anesthesia, and tissue harvesting have been described. ${ }^{11}$ The Institutional Animal Care and Use Committee of the Rhode Island Hospital approved all experiments. Animals were cared for in compliance with the Principles of Laboratory Animal Care formulated by the National Society for Medical Research and the Guide for the Care and Use of Laboratory Animals.

\section{Microvessel Studies, Coronary Angiography}

Our group has previously described detailed methods for microvessel studies and coronary angiography. ${ }^{11}$ Coronary arterioles taken from ischemic myocardium were isolated and microvascular relaxation responses were measured after being exposed to endothelium-dependent and endothelium-independent agents. Relaxation responses were defined as percent relaxation of the preconstricted (thromboxane analog U46619) diameter. Coronary angiography was performed during the final procedure to demonstrate occlusion of the LCx. A cardiologist interpreted recorded images in a blinded fashion to assess collateral formation. Collaterals were graded according to the well-validated Rentrop system of 0 to 3 . $^{5,12}$ Rentrop score is a scoring system to grade collateral filling vessels: grade 0 (no visible filling of any collateral channels), grade 1 (collateral filling branches of vessel without any dye reaching the epicardial segment of that vessel), grade 2 (partial collateral filling of the epicardial segment of the vessel being dilated), and grade 3 (complete collateral filling of the vessel being dilated).

\section{Myocardial Perfusion}

Methods for myocardial perfusion have been described at rest and during rapid cardiac pacing. ${ }^{11}$ Briefly, gold isotope-labeled microspheres (Biophysics Assay Laboratory, Worcester, Mass) were injected into the left atrium during transient LCx occlusion at the time of initial ameroid placement to determine the territory at risk. Lutetium and Europium isotope-labeled microspheres were injected at rest and with demand pacing (160 beats/min), respectively (all microspheres obtained from 
TABLE 1. Baseline microvessel diameter and percentage preconstriction

\begin{tabular}{|c|c|c|c|c|}
\hline Targets & HCC & LCI & HCI & $\begin{array}{c}P \\
\text { value }\end{array}$ \\
\hline $\begin{array}{l}\text { ADP baseline } \\
\text { diameter } \mu \mathrm{L}\end{array}$ & $132.5 \pm 41.5$ & $114.3 \pm 15.0$ & $117.6 \pm 35.8$ & .62 \\
\hline $\begin{array}{l}\mathrm{ADP} \% \\
\text { preconstriction }\end{array}$ & $32.0 \pm 10.4$ & $35.1 \pm 7.3$ & $37.1 \pm 6.8$ & .56 \\
\hline $\begin{array}{l}\text { SNP baseline } \\
\quad \text { diameter } \mu \mathrm{L}\end{array}$ & $151.3 \pm 11.1$ & $114.4 \pm 28.5$ & $116.4 \pm 29.4$ & .08 \\
\hline $\begin{array}{l}\text { SNP \% } \\
\text { preconstriction }\end{array}$ & $34.5 \pm 7.3$ & $32.2 \pm 8.8$ & $35.7 \pm 10.1$ & .75 \\
\hline \multicolumn{5}{|c|}{$\begin{array}{l}\text { Values reported } \pm \text { standard deviation compared with HCC. One-way analysis of } \\
\text { variance performed to determine } P \text { value. Bonferroni multiple comparison tests } \\
\text { were performed. } H C C \text {, High cholesterol control; } L C I \text {, low-dose calpain inhibition; } \\
H C I \text {, high-dose calpain inhibition; } A D P \text {, adenosine diphosphate; } S N P \text {, sodium } \\
\text { nitroprusside. }\end{array}$} \\
\hline
\end{tabular}

Biophysics Assay Laboratory). Tissue samples obtained after euthanasia were divided into 10 ventricular sections for isotope-labeled microsphere assays, which were exposed to neutron beams and measured with a gamma counter.

\section{Protein Expression}

Preparation of protein lysates and technique for Western blotting and band quantification have been described. ${ }^{13}$ Primary antibodies used were vascular endothelial growth factor (VEGF), vascular endothelial growth factor receptor 1 (VEGFR1), vascular endothelial growth factor receptor 2 (VEGFR2), vascular endothelial (VE) cadherin, extracellular signal-regulated kinase (ERK), phosphorylated ERK (pERK), inducible nitric oxide synthase (iNOS), endostatin, angiopoietin 1, ras homolog gene family member A (RhoA), tau, talin, paxillin, and vinculin (all from Cell Signaling, Beverly, Mass). All membranes were probed with glyceraldehyde-3-phosphate (Cell Signaling) to correct for loading error.

\section{Immunohistochemical Staining for Angiogenesis}

A detailed description for tissue preparation and immunohistochemical staining for angiogenesis has been published. ${ }^{14}$ Formalin-fixed tissue sections were incubated with antibodies against porcine endothelial marker CD-31 (R\&D Systems, Minneapolis, Minn) and smooth muscle actin (Sigma-Aldrich, St Louis, Mo) followed by appropriate Alexa-Flour conjugated antibody (Jackson ImmunoResearch, West Grove, Pa). Images were captured at $20 \times$ magnification with a Nikon E800 Eclipse microscope (Nikon, Tokyo, Japan) at the same exposure in 3 random fields. Capillaries were defined as structures 5 to $25 \mu \mathrm{m}^{2}$ in a cross-sectional area. Arterioles were defined by localization of smooth muscle actin and CD31 staining. Arteriolar and capillary density were measured using Image $\mathbf{J}$ software (National Institutes of Health, Bethesda, Md) in a blinded fashion. Representative images are shown in Figures.

\section{Data Analysis}

Analysis of variance was performed with Bonferroni corrections for multiple comparisons. We used GraphPad (GraphPad Software Inc, La Jolla, Calif) to obtain $P$ values and make all graphs. All results are expressed as fold change \pm standard error of the mean compared with the HCC group. Results in Tables 1 and 2 are expressed as fold change \pm standard deviation as compared with the HCC group.
TABLE 2. Protein expression in collateral-dependent myocardium

\begin{tabular}{lllll}
\hline \multicolumn{1}{c}{ Targets } & HCC & LCI & HCI & P value \\
\hline Angiogenesis proteins & & & \\
VEGF & $1 \pm 0.366$ & $1.96 \pm 0.819$ & $1.30 \pm 0.245$ & $.02^{*}$ \\
VEGF R1 & $1 \pm 0.208$ & $1.71 \pm 0.380$ & $1.26 \pm 0.289$ & $.0003^{*}, \ddagger$ \\
VEGF R2 & $1 \pm 0.249$ & $2.42 \pm 0.839$ & $1.19 \pm 0.244$ & $.0003^{*}, \ddagger$ \\
VE cadherin & $1 \pm 0.258$ & $1.23 \pm 0.172$ & $1.77 \pm 0.576$ & .58 \\
iNOS & $1 \pm 0.379$ & $1.01 \pm 0.344$ & $1.71 \pm 1.390$ & .21 \\
ERK & $1 \pm 0.322$ & $1.41 \pm 0.508$ & $1.33 \pm 0.794$ & .32 \\
pERK & $1 \pm 0.469$ & $1.23 \pm 0.594$ & $1.25 \pm 0.538$ & .54 \\
Endostatin & $1 \pm 0.401$ & $1.02 \pm 0.278$ & $0.97 \pm 0.484$ & .99 \\
Angiopoietin 1 & $1 \pm 0.710$ & $1.16 \pm 0.599$ & $0.90 \pm 0.479$ & .67 \\
Structural proteins & & & & \\
RhoA & $1 \pm 0.198$ & $1.08 \pm 0.305$ & $1.03 \pm 0.226$ & .21 \\
Tau & $1 \pm 0.342$ & $1.08 \pm 0.373$ & $1.06 \pm 0.372$ & .91 \\
Talin & $1 \pm 0.115$ & $1.71 \pm 0.179$ & $1.79 \pm 0.496$ & $.0002^{*}, \dagger$ \\
Paxillin & $1 \pm 0.386$ & $0.91 \pm 0.309$ & $1.47 \pm 0.296$ & .11 \\
Vinculin & $1 \pm 1.179$ & $0.65 \pm 0.424$ & $0.71 \pm 0.342$ & .60 \\
\hline
\end{tabular}

Protein expression listed as fold change \pm standard deviation compared with HCC. One-way analysis of variance performed to determine $P$ value. Bonferroni multiple comparison tests were performed. $H C C$, High cholesterol control; $L C I$, low-dose calpain inhibitor; $H C I$, high-dose calpain inhibition; $V E G F$, vascular endothelial growth factor; VEGFRI, vascular endothelial growth factor receptor 1; VEGFR2, vascular endothelial growth factor receptor 2; VE, vascular endothelial; iNOS, inducible nitric oxide synthase; $E R K$, extracellular signal-regulated kinase; $p E R K$ phosphorylated extracellular signal-regulated kinase. *Significant difference between HCC and LCI groups. †Significant difference between HCC and HCI. ‡Significant difference between LCI and HCI groups.

\section{RESULTS}

\section{Animal Model and Coronary Angiography}

All animals included for analysis survived to completion of the study. Before sacrifice, coronary catheterization was performed and demonstrated occlusion of the LCx secondary to ameroid placement in all animals. There was a trend toward increased collateral vessel formation in the HCI group versus the HCC and LCI groups, but this difference did not reach statistical

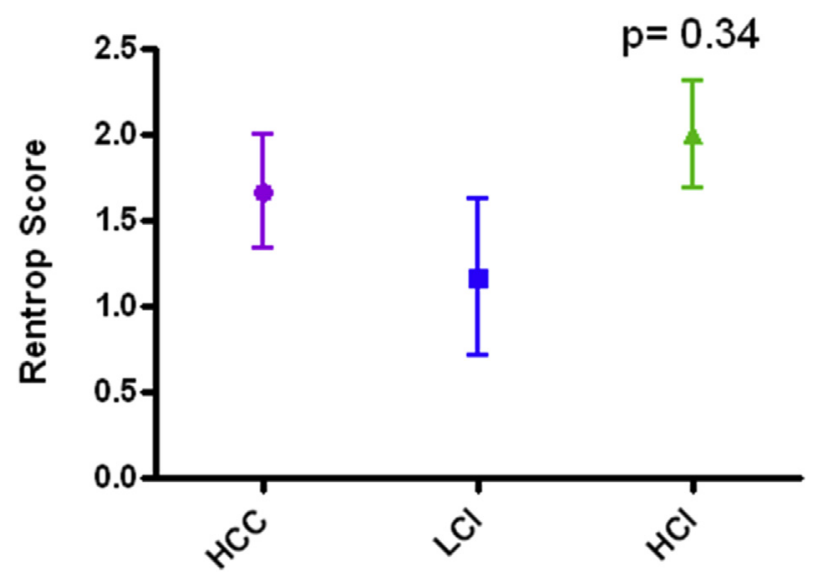

FIGURE 1. Rentrop scores interpreted from x-ray coronary angiography. HCC, High cholesterol control; LCI, low-dose calpain inhibition; $H C I$, high-dose calpain inhibition. 

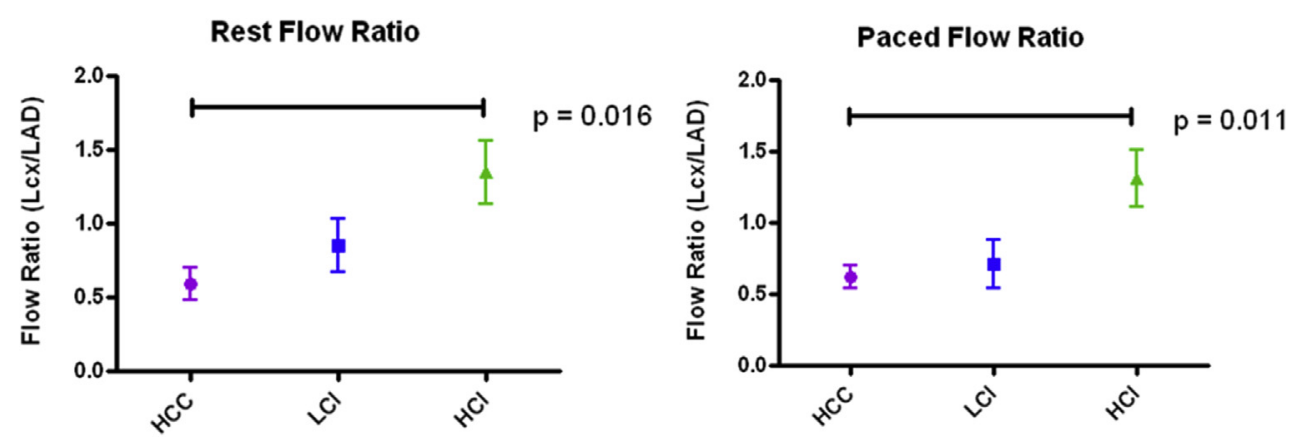

FIGURE 2. Myocardial perfusion at rest and with demand pacing. There was a significant improvement in the HCI group compared with the HCC group at rest and with demand pacing at 160 beats/min. $L C x$, Left circumflex artery; $L A D$, left anterior descending; $H C C$, high cholesterol control; $L C I$, low-dose calpain inhibition; $H C I$, high-dose calpain inhibition.

significance. Rentrop scores were as follows: HCC, $1.67 \pm 0.33 ;$ LCI, $1.17 \pm 0.45 ;$ HCI, $2.0 \pm 0.32$ $(P=.34)$ (Figure 1).

\section{Myocardial Perfusion}

Myocardial perfusion ratios (LCx/left anterior descending) were calculated at rest (HCC, $0.59 \pm 0.11$; LCI, $0.85 \pm 0.18$; HCI, $1.34 \pm 0.21$ ) and with demand pacing (HCC, $0.62 \pm 0.08$; LCI, $0.71 \pm 0.16$; HCI, $1.31 \pm 0.20$ ). When compared with the HCC group, myocardial perfusion ratios were significantly improved in the HCI group $(P=.016)$. Likewise, when the heart was stressed with ventricular demand pacing to 160 beats/min, there was a significant improvement in flow ratio in the HCI group when compared with the HCC group $(P=.011)$ (Figure 2).

\section{Microvessel Analysis}

Microvessels (100-160 $\mu \mathrm{m}$ diameter) were precontracted with the thromboxane analog U46619 by $20 \%$ to $50 \%$ of the baseline diameter. There were no significant differences in size or precontraction between groups (Table 1). When compared with the control group, microvascular endothelium-dependent relaxation was greater in the HCI group and significantly improved in the LCI group as demonstrated by the relaxation response to adenosine diphosphate $(P=.001)$. Endothelium-independent microvessel relaxation, as demonstrated by microvascular response to sodium nitroprusside, was similar among the groups $(P=.42)$. There were no differences in baseline microvessel diameter or percentage of preconstruction in the adenosine diphosphate-or sodium nitroprusside-treated microvessels (Table 1 and Figure 3).
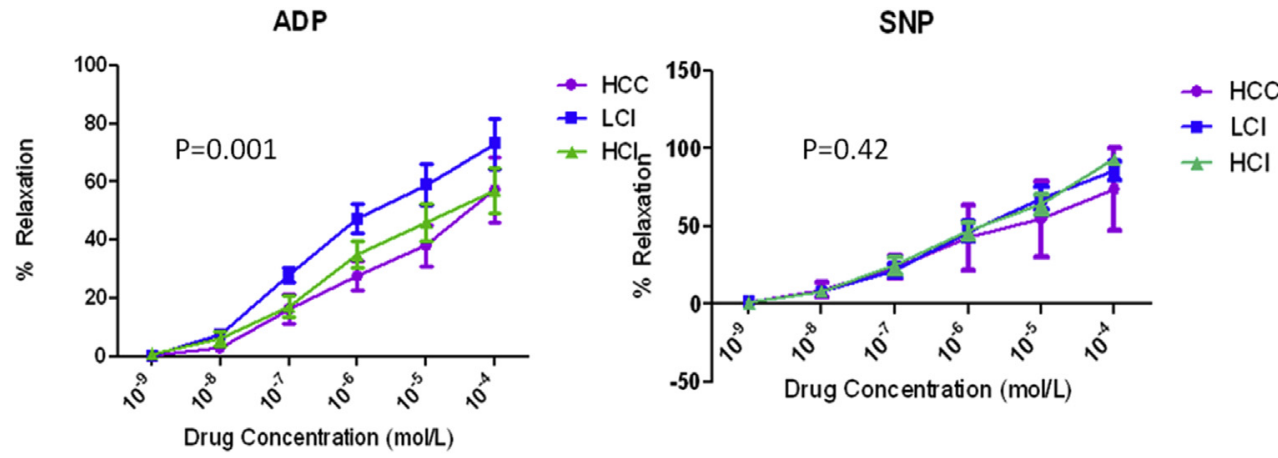

FIGURE 3. Microvessel reactivity. There was an improvement in endothelium-dependent adenosine diphosphate microvessel reactivity in the CI groups compared with the control group, with a significant improvement in the LCI group. No differences were seen in microvessel reactivity endothelium-independent (sodium nitroprusside) vasodilators in the groups. There were no differences in baseline microvessel diameter and percentage of preconstriction. No difference was seen in baseline diameter or percentage of preconstriction in adenosine diphosphate- or sodium nitroprusside-treated microvessels. Control: HCC. ADP, Adenosine diphosphate; HCC, high cholesterol control; LCI, low-dose calpain inhibition; HCI, high-dose calpain inhibition; SNP, sodium nitroprusside. 


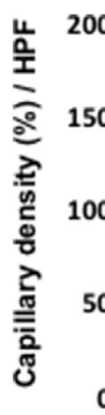

A 0
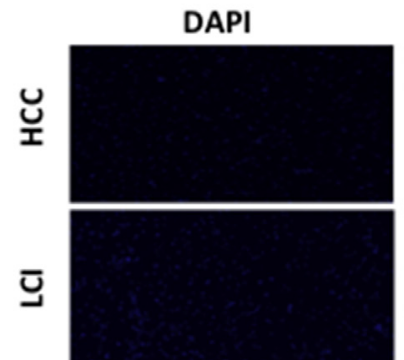

노

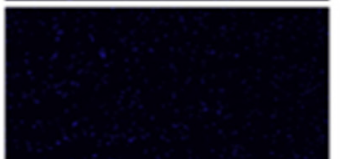

$$
p=0.01
$$

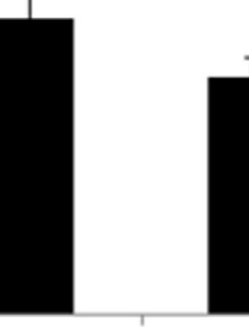

$\mathbf{L C I}$
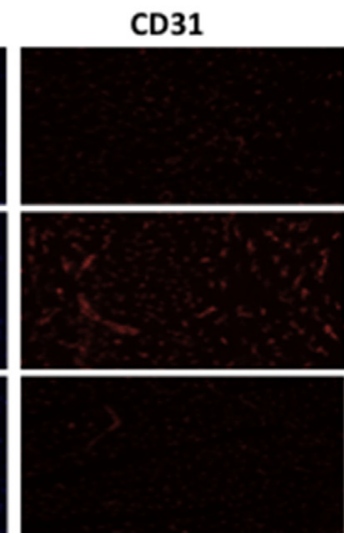

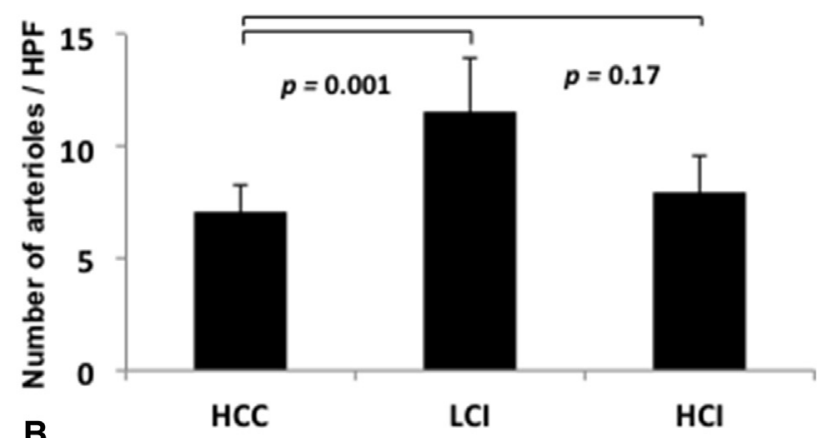

B
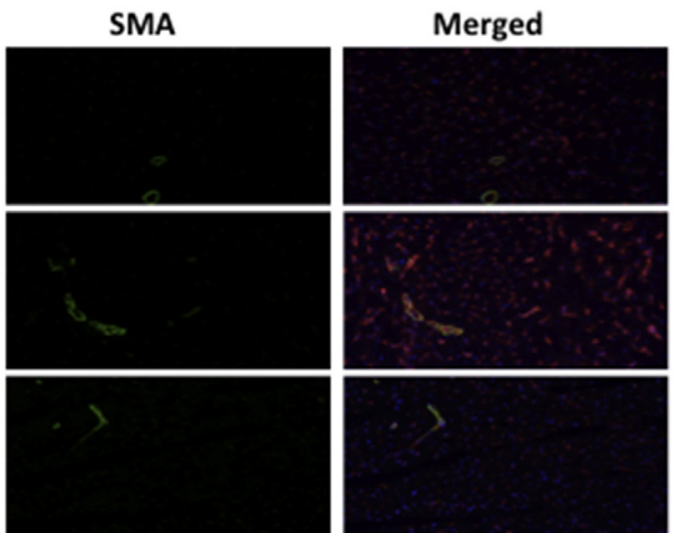

FIGURE 4. A, Endothelial cell density staining for CD31. Significant increase seen in capillary density in LCI and HCI groups compared with HCC group. B, Arteriolar cell density staining for smooth muscle actin. Significant increase in arteriolar counts in LCI group compared with control. C, Representative images in high-power field. CD31 is red, and smooth muscle actin is green. HPF, High-power field; HCC, high cholesterol control; LCI, low-dose calpain inhibition; HCI, high-dose calpain inhibition; DAPI, 4',6-diamidino-2-phenylindole; SMA, smooth muscle actin.

\section{Angiogenesis and Protein Expression}

Compared with the control group, capillary density (percentage of capillary density/high-power field) was significantly increased in the LCI group $(P=.001)$ and the HCI group $(P=.001)$ (Figure 4$)$. Arteriolar counts (number of arterioles/high-power field) were also significantly increased in the LCI group $(P=.001)$ (Figure 4$)$.

In the ischemic myocardium, there was also a significant increase in several proangiogenic proteins, including VEGF $(P=.02)$, VEGFR1 $(P=.003)$, and VEGFR2 $(P=.003)$ in
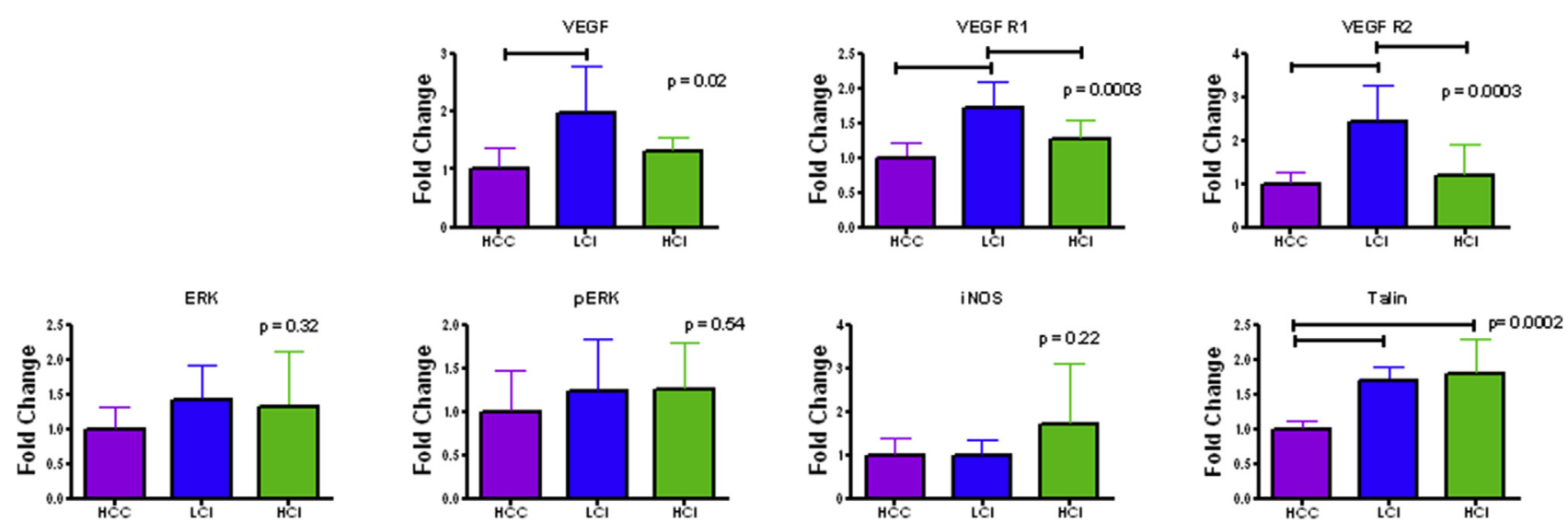

FIGURE 5. Protein expression in collateral-dependent myocardium. VEGF, Vascular endothelial growth factor; $H C C$, high cholesterol control; $L C I$, low-dose calpain inhibition; $H C I$, high-dose calpain inhibition; VEGFR1, vascular endothelial growth factor receptor 1; VEGFR2, vascular endothelial growth factor receptor 2; ERK, extracellular signal-regulated kinase; $p E R K$, phosphorylated extracellular signal-regulated kinase; iNOS, inducible nitric oxide synthase. 
the LCI group compared with the HCC group. When compared with the control group, there was a slight increase in proteins implicated in endothelial relaxation, including ERK and pERK in the LCI and HCI groups, and iNOS in the HCI group (Figure 5 and Table 2). There were no significant differences seen in the expression of vascular endothelial cadherin, endostatin, or angiopoietin-1 among the groups (Table 2). Proteins integral for focal adhesion of cellular structures were also evaluated. There was a significant increase in talin in the LCI and HCI groups compared with the HCC group $(P=.0002)$ (Figure 5 and Table 1). There were no significant differences detected in other structural proteins, including RhoA, tau, paxillin, and vinculin (Table 2).

\section{DISCUSSION}

In a hypercholesterolemic swine model of chronic myocardial ischemia, we demonstrate that CI improved proangiogenic protein expression, capillary density, and arteriolar density, and increased endothelial-dependent microvascular relaxation and myocardial blood flow.

Prior studies have demonstrated that moderate inhibition of calpain activity promotes neovascularization in the setting of hypoxia, although the exact mechanism for this remains under investigation. ${ }^{7}$ These prior studies are supported by our current study because CI resulted in an increase in capillary and arteriolar density, along with an improvement in myocardial perfusion. Data for myocardial blood flow are shown in Figure 2. There was a significant improvement in the HCI group compared with the HCC group at rest and with demand pacing at 160 beats/min, demonstrating that myocardial perfusion was increased by high-dose CI. Figure 1 shows that more animals in the HCI group demonstrated collaterals than those in the HCC or LCI group, but the increase in Rentrop score was not statistically significant. This suggests that CI may work to increase blood flow to the myocardial tissue by increasing collateral vessels to that area. The lack of significance in collateral vessel formation improvement could be the time course of the CI drug. It is possible that 5 weeks is not long enough to see these changes. This would be interesting to investigate further with more pigs in each group. Figure 4 shows that both the LCI and HCI groups had a significant increase in capillary density compared with the control HCC group. The LCI group had a significant increase in arteriole counts compared with the control. Figure 3 and Table 1 show there was an improvement in endothelium-dependent microvessel reactivity in the LCI group compared with the control. There was no difference in endothelium-independent microvessel activity among the 3 groups. This information together suggests that calpain is working to increase blood flow to the ischemic myocardial tissue by increasing vessel density in that tissue via an endothelium-dependent manner. Future work will need to evaluate the effect of $\mathrm{CI}$ in the nonischemic cardiac tissue.

Angiogenesis is an intricate process involving endothelial cell migration, proliferation, and differentiation. Figure 5 and Table 2 show that LCI and HCI demonstrated a significant increase in proteins VEGFR2, VEGFR1, and talin, whereas only LCI showed a significant increase in VEGF compared with the HCC group. VEGF is known to stimulate and regulate these processes. VEGF binds VEGFR1 and VEGFR2, which promote cell proliferation and survival by activating prosurvival proteins, like ERK, and inhibiting apoptosis. ${ }^{14,15}$ Our current results demonstrate that CI results in an upregulation in VEGF. These results suggest that CI may play a role in the regulation of VEGFmediated angiogenesis.

VEGF has been shown to induce calpain activity in endothelial cells and to paradoxically induce abnormalities in vasculature in the setting of hypoxia. ${ }^{7,8}$ These abnormalities typically result in architectural defects leading to poor blood vessel function and resulting poor blood flow. VEGF induction of calpain activity results in impairments in cytoskeletal architecture, and moderate inhibition of calpain improves these structures. ${ }^{7,8}$ Calpains are known to mediate cytoskeletal and cell adhesion through the regulation of focal adhesion proteins. Our study demonstrated that CI results in a significant upregulation in one such protein, talin. Calpain-dependent regulation of talin has been well demonstrated in other studies, ${ }^{16}$ and CI resulting in increased talin has been shown to improve microvascular contractility. ${ }^{17}$ Another study using a porcine model of right heart failure demonstrated that right ventricular pressure overload resulted in disrupted talin organization, whereas CI resulted in preservation in abundance and organization of talin, with an associated improvement in contractility. ${ }^{18}$

Of note, there was no significant difference among the 3 groups for vascular endothelial cadherin, iNOS, ERK, pERK, endostatin, angiopoietin 1, RhoA, tau, paxillin, or vinculin. These findings suggest that calpain may not have a significant effect on the ERK/ iNOS pathways and may not modulate cytoskeletal structure through upregulation of RhoA, tau, paxillin, or vinculin.

Other possible mechanisms for the beneficial effects of CI are through the prevention of calpain-induced endothelial dysfunction. A number of groups have demonstrated that hypoxia induces upregulation of calpain in endothelial cells. ${ }^{19,20}$ In a rodent model of ischemic retinopathy, Hoang and colleagues ${ }^{8}$ found that hypoxia activates calpain in endothelial cells and resulted in disruption of the actin cytoskeleton, whereas moderate inhibition of calpain 
resulted in improved vascular perfusion and improved capillary morphogenesis and organization of actin cytoskeleton. Stalker and colleagues ${ }^{20}$ reported that calpain inhibitors have anti-inflammatory effects in the setting of hyperglycemia. Other studies have demonstrated beneficial effects of $\mathrm{CI}$ with respect to circulatory failure. $^{21,22}$ In a rodent model of hemorrhagic shock, McDonald and colleagues ${ }^{21}$ demonstrated that CI reduced circulatory failure. Ruetten and Thiemermann ${ }^{22}$ demonstrated that CI attenuated circulatory failure and multiorgan dysfunction through prevention of vascular hyperactivity in a rat model of endotoxic shock. In our current study using a large animal model of chronic ischemia, we similarly demonstrate improved microvascular response in the setting of hypercholesterolemia with CI. These results taken together suggest that calpains likely play an important role in hyperglycemiainduced inflammation and microvascular dysfunction in the cardiovascular system.

We demonstrated that collateral-dependent blood flow was most improved in the HCI group, whereas microvessel relaxation, angiogenic protein expression, arteriolar density, and capillary density were most improved in the LCI group. However, we do demonstrate that some amount of moderate CI (the HCI or LCI) in the setting of hypercholesterolemia and chronic myocardial ischemia improves myocardial perfusion (as indicated by the increase in blood flow), microvascular relaxation (as indicated by the endothelium dependent microvessel reactivity study), and proangiogenic protein expression (VEGFR1, VEGFR2, and talin); however, the data presented in this study cannot be used to determine the optimal dose of CI required to improve the cardiovascular parameters in our metabolic syndrome model. It seems that the optimal dose of CI to bring about these positive effects may be in between the doses used in our LCI and HCI protocol. In other words, it is plausible that LCI and HCI are somewhere on the dose-response curve of CI where changes in dose do not have a significant change in response. Future dose-response studies are required to obtain the precise level of $\mathrm{CI}$ necessary for positive cardiovascular effects. We believe these remarks are in line with our hypothesis that a certain range and levels of CI (somewhere between LCI and HCI doses) improve myocardial blood flow.

Our study indicates that $\mathrm{CI}$ is working to increase blood flow to ischemic myocardial tissue in an endothelial-dependent manner. However, future work will be needed to identify exactly how CI is having an effect. Our laboratory has since found that moderate CI decreases tissue oxidative stress in the ischemic myocardial tissue of the same pig model. ${ }^{23}$ Our laboratory is currently studying this hypothesis, which is beyond the scope of the current study. Functional data, such as regional oxygen consumption, will be addressed in future studies.

\section{Study Limitations}

Given that this is a large animal model, we have kept the number of animals to a minimum while still allowing appropriate statistical power. As mentioned earlier, sampling error at the time of harvest can certainly affect the interpretation of the data. Further studies with larger animal groups and sample distributions would help diminish these types of errors. Although we provide important functional and molecular data as to the possible benefits of CI, the mechanisms by which these drugs exert their beneficial effects remain unclear. Furthermore, although we used 2 different doses of CI, the drug was given over a fixed period of time (for 5 weeks) and the tissue analyzed represents only 1 time point at the completion of our study. Because calpain regulates several different and dynamic pathways, the ideal dose and time for treatment require further investigation. The pigs were started on CI at 3 weeks because this is the amount of time it takes for the ameroid to fully occlude the artery. Given that this is a chronic ischemia model, we wanted to mimic a completely occluded vessel in the setting of a high-fat diet before beginning therapy. It would have been interesting to give the drug at different intervals and doses, but for the scope of this study we used the 3week time point to avoid any of the acute disturbances and ischemia that may have resulted postameroid and before having had an established high-fat diet. Moreover, the side effects of CI have not been described. Although our study was not specifically designed to evaluate the adverse effects of the drug, there were none clearly observed during the course of treatment.

\section{CONCLUSIONS}

Moderate CI in the setting of hypercholesterolemia and chronic myocardial ischemia improves proangiogenic protein expression, microvascular relaxation, and myocardial perfusion. These findings may have important clinical implications for the treatment of patients with severe CAD and microvascular dysfunction resulting from hypercholesterolemia. Perhaps the greatest limitation for studying and developing regenerative myocardial and collateral therapies in this patient population is the lack of validation in small and large animal models. Several studies have demonstrated favorable outcomes in small animal models or otherwise healthy large models. These studies often have not been clinically applicable, or relevant, because of the known microvascular, functional, and molecular differences resulting from hyperglycemia and hypercholesterolemia. ${ }^{1,4}$ These differences have been well demonstrated in clinical investigations.,4,5 $\mathrm{We}$ 
think that the use of a diseased large animal model provides a better indication of the utility of regenerative therapies, whether growth factor-, gene-, or cell-based therapies. In this study, we demonstrate that CI helps overcome some of the negative effects of hypercholesterolemia on the regenerative process and collateral vessel formation. Currently, calpain inhibitors are experimental drugs and have not been developed as therapeutic agents in patients. The results of this study are encouraging, and further studies are merited to help elucidate the mechanism and extent by which calpain inhibitors exert their beneficial effects.

\section{Conflict of Interest Statement}

Dr Sellke reports consulting fees from CSL Behring, Stryker, the Medicines Compnay, and Boehringer Ingelheim. All other authors have nothing to disclose with regard to commercial support.

\section{References}

1. Lassaletta AD, Chu LM, Sellke FW. Therapeutic neovascularization for coronary disease: current state and future prospects. Basic Res Cardiol. 2011;106: 897-909.

2. Bell MR, Gersh BJ, Schaff HV, Holmes DR Jr, Fisher LD, Alderman EL, et al Effect of completeness of revascularization on long-term outcome of patients with three-vessel disease undergoing coronary artery bypass surgery. A report from the coronary artery surgery study (CASS) registry. Circulation. 1992;86: 446-57.

3. Lawrie GM, Morris GC Jr, Silvers A, Wagner WF, Baron AE, Beltangady SS, et al. The influence of residual disease after coronary bypass on the 5-year survival rate of 1274 men with coronary artery disease. Circulation. 1982;66: 717-23.

4. Boodhwani M, Sodha NR, Mieno S, Xu SH, Feng J, Ramlawi B, et al. Functional, cellular, and molecular characterization of the angiogenic response to chronic myocardial ischemia in diabetes. Circulation. 2007;116:I31-7.

5. Abaci A, Oguzhan A, Kahraman S, Eryol NK, Unal S, Arinc H, et al. Effect of diabetes mellitus on formation of coronary collateral vessels. Circulation. 1999; 99:2239-42.

6. Franco SJ, Huttenlocher A. Regulating cell migration: calpains make the cut. $J$ Cell Sci. 2005;118:3829-38.

7. Hoang MV, Nagy JA, Fox JE, Senger DR. Moderation of calpain activity promotes neovascular integration and lumen formation during VEGF-induced pathological angiogenesis. PLoS One. 2010;5:e13612.
8. Hoang MV, Smith LE, Senger DR. Calpain inhibitors reduce retinal hypoxia in ischemic retinopathy by improving neovascular architecture and functional perfusion. Biochim Biophys Acta. 2011;1812:549-57.

9. Nakajima E, David LL, Bystrom C, Shearer TR, Azuma M. Calpain-specific proteolysis in primate retina: contribution of calpains in cell death. Invest Ophthalmol Vis Sci. 2006;47:5469-75.

10. Potter DA, Tirnauer JS, Janssen R, Croall DE, Hughes CN, Fiacco KA, et al. Calpain regulates actin remodeling during cell spreading. J Cell Biol. 1998; 141:647-62.

11. Robich MP, Osipov RM, Nezafat R, Feng J, Clements RT, Bianchi C, et al. Resveratrol improves myocardial perfusion in a swine model of hypercholesterolemia and chronic myocardial ischemia. Circulation. 2010;122:S142-9.

12. Rentrop KP, Cohen M, Blanke H, Phillips RA. Changes in collateral channel filling immediately after controlled coronary artery occlusion by an angioplasty balloon in human subjects. J Am Coll Cardiol. 1985;5:587-92.

13. Sabe AA, Elmadhun NY, Sadek AA, Chu LM, Bianchi C, Sellke FW. Differential effects of atorvastatin on autophagy in ischemic and nonischemic myocardium in Ossabaw swine with metabolic syndrome. J Thorac Cardiovasc Surg. 2014;148: 3172-8.

14. Elmadhun NY, Lassaletta AD, Chu LM, Liu Y, Feng J, Sellke FW. Atorvastatin increases oxidative stress and modulates angiogenesis in Ossabaw swine with the metabolic syndrome. J Thorac Cardiovasc Surg. 2012;144:1486-93.

15. Ferrara N, Gerber HP, LeCouter J. The biology of VEGF and its receptors. Nat Med. 2003;9:669-76.

16. Franco SJ, Rodgers MA, Perrin BJ, Han J, Bennin DA, Critchley DR, et al. Calpain-mediated proteolysis of talin regulates adhesion dynamics. Nat Cell Biol. 2004;6:977-83.

17. Kotecki M, Zeiger AS, Van Vliet KJ, Herman IM. Calpain- and talin-dependent control of microvascular pericyte contractility and cellular stiffness. Microvasc Res. 2010;80:339-48.

18. Ahmad HA, Lu L, Ye S, Schwartz GG, Greyson CR. Calpain inhibition preserves talin and attenuates right heart failure in acute pulmonary hypertension. Am J Respir Cell Mol Biol. 2012;47:379-86.

19. Su Y, Block ER. Role of calpain in hypoxic inhibition of nitric oxide synthase activity in pulmonary endothelial cells. Am J Physiol. 2000;278:L1204-12.

20. Stalker TJ, Skvarka CB, Scalia R. A novel role for calpains in the endothelial dysfunction of hyperglycemia. FASEB J. 2003;17:1511-3.

21. McDonald MC, Mota-Filipe H, Paul A, Cuzzocrea S, Abdelrahman M, Harwood S, et al. Calpain inhibitor I reduces the activation of nuclear factor-kappaB and organ injury/dysfunction in hemorrhagic shock. FASEB J. 2001; 15:171-86.

22. Ruetten H, Thiemermann C. Effect of calpain inhibitor I, an inhibitor of the proteolysis of I kappa B, on the circulatory failure and multiple organ dysfunction caused by endotoxin in the rat. Br J Pharmacol. 1997;121:695-704.

23. Potz BA, Sabe AA, Elmadhun NY, Feng J, Lui Y, Mitchell H, et al. Calpain inhibition decreases apoptosis in a swine model of chronic myocardial ischemia. Surgery. 2015; 158:445-52.

Key Words: angiogenesis, animal model surgery, calpain inhibition, collateral circulation, ischemic heart disease, myocardial revascularization, perfusion 